2. J. Eells, Jr., On the geometry of function spaces, International Symposium on Algebraic Topology, pp. 303-308, Universidad Nacional Autonoma de México and UNESCO, Mexico City, 1958.

3. S. Lang, Introduction to differential manifolds, Interscience, New York, 1962.

4. C. B. Morrey, Existence and differentiability theorems for variational problems for multiple integrals, Partial Differential Equations and Continuum Mechanics, Univ. of Wisconsin Press, Madison, Wis., 1961.

5. - Multiple integral problems in the calculus of variations and related topics, Ann. Scuola Norm. Sup. Pisa 14 (1960), 1-61.

6. M. Morse, The calculus of variations in the large, Amer. Math. Soc. Colloq. Publ. Vol. 18, Amer. Math. Soc., Providence, R. I., 1934.

7. J. Moser, On the regularity problem for elliptic and parabolic differential equations, Partial Differential Equations and Continuum Mechanics, Univ. of Wisconsin Press, Madison, Wis., 1961.

8. L. Nirenberg, On elliptic partial differential equations, Ann. Scuola Norm Sup. Pisa 13 (1959), 115-162.

BRANDEIS UNIVERSITY AND Columbia University

\title{
ON THE SOLUTIONS OF THE WAVE EQUATION IN A QUADRANT OF $R^{4}$
}

\author{
BY Y. W. CHEN $^{1}$ \\ Communicated by Peter D. Lax, August 21, 1963
}

The problem of Goursat in its various forms has been studied extensively for hyperbolic differential equations in two independent variables, while similar questions for the equations with more than two variables have received much less attention than they properly deserve. One finds simple problems which do not admit any solution, although the same problems in two dimensions are well proposed. Other examples show that solutions with "correct" data may produce a curve of singularity in the interior. ${ }^{2}$ In this paper we study solutions of the wave equation

$$
w_{x x}+w_{x_{1}^{\prime} x_{1}^{\prime}}+w_{x_{2^{\prime} x_{2}^{\prime}}}-w_{t t}=0
$$

in the quarter-space

$$
Q^{4}:|t|<x, x>0,-\infty<x^{\prime}<\infty,\left(x^{\prime}=\left(x_{1}^{\prime}, x_{2}^{\prime}\right)\right),
$$

1 This research was supported by the Air Force office of Scientific Research. Part of the work was done while the author was on a sabbatical leave from Wayne State University in 1961-1962.

2 One of the examples was communicated to the author by F. John. That the problem with $w(X, 0)=0$ and arbitrary values on $C_{+}$may have no solution was pointed out by H. Lewy in a discussion. To both of them the author is greatly indebted. 
bounded by the characteristics $C_{+}$and $C_{-}$respectively given by $x=t$ and $x=-t, x>0,-\infty<x^{\prime}<\infty$. As the plane of symmetry we have the initial $(t=0)$ half-space $H^{3}: x>0,-\infty<x^{\prime}<\infty$. Our main result is, in brief, that a solution in $Q^{4}$ is uniquely determined by its values on one of the characteristics, provided the solution satisfies a mild regularity condition at infinity on $H^{3}$. This result is in contrast to the two dimensional theory where values of the solution on both $C_{+}$and $C_{-}$, or, on $H^{3}$ and one of the characteristics, can be prescribed.

The basic relation between the values of the solutions on $C_{+}\left(C_{-}\right)$ and the data on $H^{3}$ is that given by the Poisson formula. Let $X=\left(x, x^{\prime}\right)$, and denote by $S M(f ; X, \rho)$ the spherical mean of $f$ over the sphere with center $X$ and radius $\rho$. Then for functions which are continuous in the half-space $R_{+}^{3}\left(x>0,-\infty<x^{\prime}<\infty\right)$ and on its boundary $x=0$, one can form the mean $S M^{*} f=S M(f ; X, x)$ in which the $x$ coordinate of the center is also the radius of the sphere. A moment of reflection will convince the reader that the key question in our problem is the following: What is the necessary and sufficient condition for a function $\phi$ in order that $\phi=S M^{*} f$ holds for some $f$ in $R_{+}^{3}$ ? The analysis of this question and its answer (see Theorem 3 ) lead to the study of the classes $\mathfrak{f}^{\prime}$ and $\mathfrak{f}^{\prime}$ of solutions in $Q^{4}$, which can be represented by the weighted parabolic means ${ }^{3}$ of their boundary values on $C_{-}$. Their values on $C_{+}$are then uniquely determined. These solutions have some very interesting properties (see $\$ 1$ ). It will be shown that a solution which is given by Poisson's formula and is "regular" at infinity on $H^{3}$, belongs to $\mathfrak{f}^{\prime}$. From this follows the uniqueness theorem in $\S 2$. A well-proposed Goursat problem is given in $\$ 3 .{ }^{4}$

A number of identities for the iterated spherical and parabolical means ${ }^{5}$ are established in this paper. They are needed for the uniqueness proof, and they serve as an important tool for inverting the operators such as the one given by $\phi=S M^{*} f$. While the method used to establish these identities is the same, the geometry involved is different in each case.

1. The class $t$ of solutions by the parabolic means.

Notations. $p(X, s)$ denotes the paraboloid in $R_{+}^{3}$ described by all the points $Y$ satisfying $(y-x)^{2}+\left|y^{\prime}-x^{\prime}\right|^{2}=(y+s)^{2}$, for any fixed

3 These are weighted mean values of functions over paraboloids with cylindrical symmetry, see $\$ 1$.

${ }^{4}$ Details of the proofs in this work will be presented elsewhere.

${ }^{5}$ For the identity on iterated spherical means and its applications see [1]. Added in proof: Several of the identities used here can be proved simply by observing that both sides represent the same solution of the wave equation because of the same initial conditions at $s=0$. 
$s$ restricted to $-x<s \leqq x$. Let the distance of a point $Y$ to the axis of symmetry be $r(x, y, s)=\sqrt{ }((2 y-x+s)(x+s))$. The following mean value over $p(X, s)$ is formed:

$$
P M(\zeta ; X, s)=\frac{1}{x+s} \frac{1}{2 \pi} \int_{b}^{\infty} \int_{0}^{2 \pi} \zeta\left(y, x^{\prime}+r(x, y, s) \theta^{\prime}\right) d \theta d y
$$

with $b=(x-s) / 2, \theta^{\prime}=(\cos \theta, \sin \theta)$. A function $\zeta(X)$ continuous in $R_{+}^{3}$, is said to be $p$-integrable, if the above integral is absolutely convergent for arbitrary $(X, s)$ and for $s=-x$ (when $r=0$ ). We shall also denote the above integral in brief by $P M^{8} \zeta$ for $-x<s<x$, specifically $P M^{0} \zeta$ for $s=0$, and set $P M^{*} \zeta=P M(\zeta ; X, x)$. Furthermore let $\delta \zeta=\partial(x \zeta(X)) / \partial x$. Finally we introduce the important operator $E \zeta=2 P M^{*}(\delta \zeta)$. The following lemma is basic:

Lemma 1. Let $\zeta \in C_{1}, \zeta, \delta \zeta$ p-integrable. We have the identity:

$$
(x+s) P M(E \zeta ; X, s)=(x-s) P M(\zeta ; X,-s)
$$

for $-x<s<x$.

Note the left-hand side is an iterated mean $P M^{*}\left(P M^{*}\right)$. The geometry is that the family of paraboloids $p(Y, y)$ with $Y$ varying along $p(X, s)$ has $p(X,-s)$ as an envelope. When $s=0$, Lemma 1 shows in particular $P M^{0}(E \zeta)=P M^{0} \zeta$.

Lemma 2. Let $\zeta \in C_{2}, \zeta$, $\delta \zeta$ and $\delta^{2} \zeta$ p-integrable. Then $E E \zeta=\zeta$.

For the proof one makes use of $P M^{*}(\delta \zeta)=-\delta P M^{*} \zeta$ to write

$$
E \zeta=-\frac{\partial}{\partial x}\left(\lim _{s \rightarrow x}(x+s) P M^{\bullet} \zeta\right) .
$$

Now replace $\zeta$ by $E \zeta$ and apply Lemma 1 .

There is an unique decomposition of any $\zeta$ of Lemma $2, \zeta=\phi+\psi$, such that $\phi=E \phi$ and $\psi=-E \psi$. We shall call $\phi$ the even and $\psi$ the odd component of $\zeta . \zeta=0$ if and only if $\phi=\psi=0$.

By a simple calculation one proves

Lemma 3. Let $\zeta$ be a class $C_{1}$ in $R_{+}^{3}$, such that $\zeta$ and $x^{\prime} \cdot \partial \zeta(X) / d x^{\prime}$ are $p$-integrable and that $\nabla^{\prime}(x+t) P M(\zeta ; X, t)$ exists, where $\nabla^{\prime}$ is the Laplacian in $\left(x_{1}^{\prime}, x_{2}^{\prime}\right)$. Then the function $(x+t) P M(\zeta ; X, t)$ is a solution of the wave equation in $Q^{4}$.

Definition 1. $\mathfrak{t}$ consists of all the solutions in $Q^{4}$ given by $w(x, t)$ $=(x+t) P M(\delta \zeta ; X, t)$, where $\zeta \in C_{2}, \zeta, \delta \zeta, \delta^{2} \zeta, x^{\prime} \cdot \partial \zeta(X) / \partial x^{\prime}$ and $x^{\prime} \cdot \partial(\delta \zeta) / \partial x^{\prime}$ are $p$-integrable, and $w(x, t)$ admits the Laplacian $\nabla^{\prime}$.

It is easily seen that the boundary value of $w$ on $C_{-}(t=-x)$ is given 
by $-x \zeta(X)$, while its value on $C_{+}(t=x)$ by $x E \zeta(X)$. Because of Lemma 2, the relationship between the two functions of boundary values on $C_{-}$and $C_{+}$is a symmetrical one.

ThEOREM 1. Each element $w$ in is uniquely determined by its boundary values on one of the two characteristics $C_{+}$and $C_{-}$. To each w there are two elements in $\mathfrak{f}, u$ and $v, u(X, t)=-u(X,-t), v(X, t)=v(X,-t)$, such that $w=u+v$, and the decomposition is unique.

For $w=(x+t) P M(\delta \zeta ; X, t)$ one has $u=(x+t) P M(\delta \phi ; X, t)$ and $v=(x+t) P M(\delta \psi ; X, t)$ where $\phi$ and $\psi$ are respectively the even and odd components of $\zeta$.

\section{Uniqueness.}

Notations. The following weighted means are introduced:

$$
P M_{k}(\zeta ; X, s)=\frac{1}{2 \pi} \int_{b}^{\infty} \int_{0}^{2 \pi}(y+s)^{-k} \zeta\left(y, x^{\prime}+r(x, y, s) \theta^{\prime}\right) d \theta d y
$$

for $k=1,2$, with $b$ and $r$ being the same as in $\S 1$. Form the operators

$$
\begin{aligned}
W(\zeta) & =W(\zeta ; X, t) \\
& =-(x+t)\left[P M_{2}(\zeta ; X, t)+\frac{\partial}{\partial t} P M_{1}(\zeta ; X, t)\right],
\end{aligned}
$$

and

$$
\mathcal{E}(\zeta)=\mathcal{E}(\zeta ; X)=\lim _{t \rightarrow x} W(\zeta ; X, t) .
$$

Denote by $\bar{h}$ the product $x \cdot h(X)$ of any $h(X)$ with $x$.

It is easily verified by differentiation under the integration sign of $P M_{1}$, that $(x+t) P M(\delta \zeta ; X, t)=W(\bar{\zeta} ; X, t)$. A solution of the form $W(\zeta)$ is said to belong to the class $\mathfrak{f}^{\prime}$, so that every element in $\mathfrak{t}$ is also in $\mathfrak{t}^{\prime}$. However, to form $W(\zeta)$, less stringent conditions on the integrability of $\zeta$ are required. We shall not discuss $\mathfrak{l}^{\prime}$ here, since our interest is concerned with the uniqueness of the solutions in $Q^{4}$.

Definition 2. A solution $w(X, t)$ in $Q^{4}$ is regular if $w \in C_{2}$, and on $H^{3}$ (where $t=0$ ) $w$ and its first derivatives $w_{X}$ and $w_{t}$ are continuous at $x=0$ and are $O|X|^{-1-\epsilon}, \epsilon>0$, for large $|X|$.

TheOREM 2. A regular solution in $Q^{4}$ is identically zero if it vanishes on $C_{+}$or on $C_{-}$.

Set $w_{t}(X, 0)=f(X)$ and $w(X, 0)=g(X)$. The main task of the proof is to write the Poisson formula in terms of $W$. For this purpose we need the following two identities: First, 


$$
W\left(x S M^{*} f ; X, s\right)=s S M(f ; X, s) \quad \text { for }-x<s<x .
$$

And secondly, denoting

$$
\begin{aligned}
I(g) & =\lim _{t \rightarrow x} \frac{\partial}{\partial t} t S M(g ; X, t), \\
\frac{\partial}{\partial s} S S M(g ; X, s) & =-W(I(g) ; X, s) .
\end{aligned}
$$

Suppose we have established these identities, and let us denote for the moment $x S M^{*} f=\alpha(X)$ and $I(g)=\beta(X)$, then by passing to the limit $s \rightarrow x$ we find

$$
\mathcal{E}(\alpha)=\alpha, \quad \mathcal{E}(\beta)=-\beta .
$$

Now by assumption $w$ vanishes on $C_{+}$, so $\alpha+\beta=0$, hence $\varepsilon(\alpha+\beta)$ $=\alpha-\beta=0$. Consequently $\alpha=\beta=0, W(\alpha)=W(\beta)=0$ and $w=0$ in $Q^{4}$.

To prove the first of the above two identities one writes the iterated mean $P M_{1}\left(x S M^{*} f\right)$ as an integral over the exterior domain of the sphere $C(X,|s|)$ in $R_{+}^{3}, X$ being the center, $|s|$ the radius of the sphere. ${ }^{6}$ Differentiation of the integral with respect to $s$ leads to two terms, one of which cancels out with the first term involving $P M$ in $W$, the remaining term gives the desired result. The second identity is proved by using some of the equations derived in Theorem 3 .

If an assumption is made on the regularity property of the second derivatives of $w(X, t)$ in $H^{3}$, then such a solution will belong to $\mathfrak{f}$. Indeed, if $f$ and $g$ are the same as above, then $w=u+v$ with $u=(x+t) P M\left(\delta S M^{*} f ; X, t\right)$ and $v=-(x+t) P M\left(S M^{*}(L g) ; X, t\right),{ }^{7}$ where the operators $L=x \nabla_{x, x^{\prime}}^{2}+\partial / \partial x$.

3. A Goursat problem. A function in $R_{+}^{3}$ is said to be belong to $C_{2 \epsilon}^{\gamma}$ if it belongs to $C_{2}^{\gamma}$ for $x>0$, continuous at $x=0$ and is $O|X|^{-1-\epsilon}$, $0<\epsilon<1$, for large $|X|$.

Let $f \in C_{2 \epsilon}^{\gamma}$. If $\phi=S M^{*} f$, then also $\phi \in C_{2 \epsilon}^{\gamma}$. Form the function

$$
\Phi(X)=x P M(\phi ; X, 0) .
$$

In order to have $\Phi$ expressed in terms of $f$, one derives the following identity:

- $C(X,|s|)$ is the envelope of the family of spheres $C(Y, y)$ with their centers $Y$ varying on the paraboloid $p(X, s)$. When $s>0$, all such spheres touch $C(X, s)$ from the exterior. When $s<0, C(X,|s|)$ is contained in every $C(Y, y)$. When $s=0$ all the spheres pass through the point $X$.

' One can verify $(\partial / \partial x) I(g)=S M^{*}(L g)$. 


$$
x P M\left(S M^{*} f ; X, 0\right)=\frac{1}{2 \pi} \int f(Y) K\left(x, y, y^{\prime}-x^{\prime}\right) d Y
$$

where $K=\left((y-x)^{2}+\left|y^{\prime}-x^{\prime}\right|^{2}\right)^{-1 / 2}\left((y+x)^{2}+\left|y^{\prime}-x^{\prime}\right|^{2}\right)^{-1 / 2}$, and the domain of integration is $R_{+}^{3}$. It then follows that $\Phi \in C_{4}^{\gamma}$, and with the same operator $L$ as given above, $L \Phi=-f$. Consequently, $S M^{*}(L \Phi)$ $=-\phi$.

THEOREM 3. The following conditions for $\phi$ are necessary and suffcient in order that $\phi=S M^{*} f$ holds for some $f \in C_{2 a}^{\gamma}: \phi \in C_{2 \epsilon}^{\gamma}, \Phi \in C_{4}^{\gamma}$, $L \Phi \in C_{2 \epsilon}^{\gamma}$ and $W(\bar{\phi} ; X, s) \rightarrow \pm \bar{\phi}$ as $s \rightarrow \pm x$. (The last condition can be put in the form $E \phi=\phi$, if the first derivatives of $f$ and $\phi$ are "regular" at infinity.)

For the proof of Theorem 3 one simplifies the form of the operators $S M^{*}(L \Phi)$ and $S M(L \Phi ; X, s)$, by showing that, for any $\phi$ and $\Phi=x P M^{0} \phi$,

$$
S M(L \Phi ; X, s)=-\frac{1}{2 s}[W(\bar{\phi} ; X, s)-W(\bar{\phi} ; X,-s)]
$$

holds. In the process the following identity is used, ${ }^{8}$

$$
S M\left(x P M^{0} \phi ; X, s\right)=\frac{1}{2 s} \int_{-8}^{s}(x+\tau) P M(\phi ; X, \tau) d \tau .
$$

It is clear that the Goursat problem with $w=0$ on $H^{3}$ and $w=\Phi$ on $C_{+}$has a solution if and only if $\phi$ satisfies the conditions given above. However, if instead of $\phi$ the function $\Phi(X)=x P M(\phi ; X, 0)$ is assigned, then the problem is well-proposed. We state

TheORem 4. Let $g(X)$ and $\Phi(X)$ be given in $R_{+}^{3}$ such that $g \in C_{3}^{\prime}$ $\Phi \in C_{4}^{\gamma}, L g$ and $L \Phi$ both are $O|X|^{-1-\epsilon}$ at infinity. Then there exists one and only one solution $w(X, t)$ in $Q^{4}$, of class $C_{3}$, with $w=g$ on $H^{3}$, $P M^{0} \bar{\phi}^{-}=\Phi$ where $\bar{\phi}$ is the value of $w$ on $C_{+}$, and $w_{t}(X, 0) \in C_{2 e}^{\gamma}$.

\section{REFERENCE}

1. F. John, Plane waves and spherical means applied to partial differential equations, Interscience, New York, 1955.

\section{Wayne State University}

${ }^{8}$ The family of paraboloids $p(Y, 0)$ with the focii $Y$ varying on the sphere $C(X, s)$ has as their envelope, the two paraboloids $p(X, s)$ and $p(X,-s)$. 